\title{
Acolhimento aos pacientes e familiares atendidos no ambulatório de oncologia: um relato de experiência
}

\author{
Reception to patients and families served in clinic for oncology: \\ an experience report
}

\author{
Lucimere Maria dos Santos ${ }^{1}$ Wbiratan de Lima Souza ${ }^{2}$ Geisiane de Souza Santos ${ }^{3}$ Eliane Ramos Pereira ${ }^{4}$. \\ Rose Mary Costa Rosa Andrade Silva ${ }^{5}$ Cristina Lavoyer Escudeiro ${ }^{6}$
}

\begin{abstract}
RESUMO
O objetivo deste estudo é relatar as experiências vivenciadas por uma enfermeira e sua equipe, referente ao atendimento prestado aos pacientes e seus familiares em um ambulatório de oncologia clínica de um hospital especializado na cidade do Rio de Janeiro. Trata-se de relato de experiência vivenciado no decorrer das práticas profissionais. O primeiro contato do paciente e seus familiares no ambulatório acontece na recepção, no momento da entrega do cartão de consulta ou dos encaminhamentos, para os pacientes de primeira vez. Quando o paciente chega à recepção com qualquer queixa, imediatamente a equipe de enfermagem recebe a informação da necessidade do mesmo e realiza o atendimento, onde são aferidos os sinais vitais e o enfermeiro realiza a avaliação a partir exame físico associado ao diálogo, a fim de identificar as necessidades de saúde e a prioridade para o atendimento médico a partir das queixas descritas pelo paciente e/ou familiar. Concluí-se que experiências vivenciadas no ambulatório de oncologia clínica contribuíram de formas satisfatórias para compreensão de gerenciar os serviços e as ações da equipe de enfermagem, no que se refere acolhimento aos pacientes e familiares, bem como para tomada de decisão.
\end{abstract}

Palavras-chave: Humanização da Assistência; Neoplasias; Cuidados de Enfermagem.

\begin{abstract}
The goal of this study is to describe experiences lived by a nurse and her team, in reference to the assistance provided to the patients and their families at an oncology ambulatory, on a specialized hospital in Rio de Janeiro city. It's a report about the experiences lived in the course of professional practices. The first contact from patients and their families with the ambulatory begins at the reception, the moment of delivery the consult card or the medical referrals for the first-way patients. When the patient arrives with any complaint, the nursing team immediately receive the information of the patient needs and do the assistance, where the vital signs are checked, the nurse do a physical assessment associated to dialogue in order to identify the health needs and priorities to the medical care from the complaints reported by the patient and/or a family member. Conclusion: The experiences lived at the oncology ambulatory helped satisfactorily the understanding to manage the services and actions of the nursing team, when referencing to the patients and family reception, as like the decision make.
\end{abstract}

Keywords: Attendance Humanization; Neoplasms; Nursing Care.

${ }^{1}$ Enfermeira. Mestra do Programa de Mestrado Profissional em Enfermagem Assistencial (MPEA) da Escola de Enfermagem Aurora de Afonso Costa (EEAAC) da Universidade Federal Fluminense (UFF). Enfermeira do Instituto Nacional do Câncer (INCA - Ambulatório de Oncologia Clínica e Hematologia). Duque de Caxias - RJ, Brasil. E-mail: lucimere_santos@hotmail.com.

2 Enfermeiro. Mestrando do Programa de Mestrado Profissional em Enfermagem Assistencial (MPEA) da Escola de Enfermagem Aurora de Afonso Costa (EEAAC) da Universidade Federal Fluminense (UFF). Professor do Centro Universitário Tiradentes (UNIT-Maceió). Professor da Universidade Estadual de Ciências da Saúde de Alagoas (UNCISAL). Professor substituto da Universidade Federal de Alagoas (UFAL-Maceió). Enfermeiro Assistencial do Hospital de Emergência Dr. Daniel Houly (HEDH). Membro do Núcleo de Estudo e Pesquisa em Cidadania e Gerência e Enfermagem (NECIGEN). Maceió - AL, Brasil. E-mail: wbiratansouza@yahoo.com.br.

${ }^{3}$ Enfermeira. Mestranda do Programa de Mestrado Profissional em Enfermagem Assistencial (MPEA) da Escola de Enfermagem Aurora de Afonso Costa (EEAAC) da Universidade Federal Fluminense (UFF). Enfermeira do Hospital Estadual Roberto Chabo. Membro do Núcleo de Estudo e Pesquisa em Cidadania e Gerência e Enfermagem (NECIGEN). Saquarema - RJ, Brasil. E-mail: grisianesantos10@hotmail.com.

${ }^{4}$ Enfermeira. Pós-Doutorado em Enfermagem. Coordenadora e Docente do Programa de Mestrado Profissional em Enfermagem Assistencial (MPEA), Professora do Curso de Graduação (EEAAC/UFF). São Gonçalo - RJ. Brasil. E-mail: elianeramos.uff@gmail.com.

${ }^{5}$ Professora Dra ${ }^{a}$, Universidade Federal Fluminense, Escola de Enfermagem Aurora de Afonso Costa, Departamento de Enfermagem Médico- Cirúrgica, Programa de PósGraduação Mestrado Profissional em Enfermagem Assistencial. Niterói - RJ, Brasil. E-mail: roserosauff@gmail.com

${ }^{6}$ Enfermeira. Doutora em Enfermagem. Coordenadora do curso de Graduação em Enfermagem da Escola de Enfermagem Aurora de Afonso Costa (EEAAC) da Universidade Federal Fluminense (UFF). Docente Permanente do MPEA (EEAAC/UFF). Membro do NECIGEN. Niterói - RJ. Brasil. E-mail: cristinalescudeiro@gmail.com. 


\section{INTRODUÇÃO}

$\mathrm{O}$ acolhimento como ato ou efeito de acolher expressa, em suas várias definições, uma ação de aproximação, um "estar com" e um "estar perto de". Essa atitude implica estar em relação com algo ou alguém. É exatamente nesse sentido, de ação de "estar com" ou "estar perto de", que o acolhimento se afirma como uma das diretrizes de maior relevância ética/estética/política da Política Nacional de Humanização do SUS. Sendo este, um modo de operar os processos de trabalho em saúde, de forma a atender a todos que procuram os serviços de saúde, ouvindo seus pedidos e assumindo no serviço uma postura capaz de acolher, escutar e dar respostas mais adequadas aos usuários. ${ }^{1}$

Com a elevada expectativa de vida da população brasileira e o aumento do número de idosos em nosso país, observa-se também a elevação dos índices de doenças crônicas degenerativas e, dentre estas, o câncer, que vêm ganhando importância no perfil epidemiológico tornandose um problema de saúde pública, haja vista o número de novos casos, que vem sendo diagnosticados no país e no mundo. $^{2}$

O número estimado para 2014/2015 de acordo com o Instituto Nacional de Câncer (INCA) é de aproximadamente 576 mil novos casos de câncer no Brasil, incluindo os casos de pele não melanoma, que é o tipo mais incidente para ambos os sexos (182 mil casos novos), seguido de próstata (69 mil), mama feminina (75 mil), cólon e reto (33 mil), pulmão (27 mil), estômago (20 mil) e colo do útero (15 mil). Duas tendências importantes foram apontadas: a redução na incidência dos casos novos de câncer do colo do útero e de pulmão em homens. Esses resultados estão relacionados as iniciativas para prevenção e detecção precoce do câncer do colo do útero e das ações de prevenção ao tabagismo no Brasil. ${ }^{2}$

O INCA é um centro de alta complexidade de referência no atendimento a paciente com diagnóstico de neoplasia maligna situado no estado do Rio de Janeiro.

O hospital possui diversos setores para atendimento hospitalar, desde o atendimento emergencial, internações, serviços de cuidados paliativos até as consultas ambulatoriais. Os ambulatórios são distribuídos pelas áreas internas e externas da instituição que se dividem de acordo com os tipos de câncer como exemplo: câncer de cabeça e pescoço, câncer de bexiga, câncer de próstata e etc, obedecendo as especificidades e a modalidade de tratamento.

A entrada do paciente na instituição para a primeira avaliação acontece através do Sistema de Regulação de Vagas (SISREG) que é definido como um Sistema online desenvolvido pelo Departamento de Informática do Sistema Único de Saúde do Ministério da Saúde. O sistema é um software, disponibilizado pelo Ministério da Saúde para o gerenciamento de todo Complexo Regulatório, que vai da rede básica de saúde à internação hospitalar, visando a humanização dos serviços e maior controle do fluxo. ${ }^{3}$
Após a avaliação realizada pelo médico na primeira consulta, o paciente pode abrir matrícula no respectivo ambulatório ou ser encaminhado para o setor de triagem que após avaliação médica o mesmo pode ser conduzido para o ambulatório de oncologia clínica e assim abrir matrícula.

O fluxo de atendimento no referido ambulatório acontece de duas formas: através de um encaminhamento médico aos pacientes já matriculados na instituição, submetidos ou não ao tratamento cirúrgico e os que necessitam dar continuidade ao tratamento fazendo uso de quimioterapias (método que utiliza compostos químicos, no tratamento de doenças causadas por agentes biológicos) ou radioterapias (é o emprego de feixes de radiações ionizantes para destruir células tumorais) ou ambas, sendo empregada concomitantemente.

O câncer é uma das doenças crônicas mais temidas pelas pessoas e se caracteriza como um conjunto de sentimentos carregados de significados. O diagnóstico do câncer promove nos pacientes e seus familiares, uma sensação de catástrofe, desordem, desespero e muito sofrimento, pois o que percebemos é a relação do diagnóstico com o medo da morte, além de todas as comorbidades advindas da doença, bem como do tratamento, que promove várias reações adversas. ${ }^{4-6}$

O paciente com câncer não deve ser avaliado, somente, como mais um caso. Nesse aspecto, precisa ser empreendida uma visão holística, procurando compreendê-lo nas suas várias relações, proporcionando uma abordagem profissional humanizada e profundamente solidária, geradora não só de saúde, mas, principalmente, de vida. ${ }^{7}$

O entendimento da saúde como condição para a cidadania sugere a dinamização de ações que potencializam o bem-estar e a qualidade de vida das pessoas. Nesta perspectiva, estruturar serviços e ações em saúde que vão ao encontro das necessidades das pessoas, torna-se cada vez mais um desafio, para enfermagem, pois a medida que os determinantes sociais, biológicos, políticos, culturais, entre outros, interagem com complexidade crescente, trazendo repercussões, não apenas de imediato, mas em longo prazo. ${ }^{8}$

A enfermagem se torna imprescindível, no que se refere à promoção da assistência, a fim de identificar as necessidades dos pacientes e seus familiares, oferecendolhes um atendimento humanizado e acolhedor.

A sala de espera de uma unidade ambulatorial deve ser vista, pelos profissionais de saúde, como um espaço propício para promover, o diálogo, acolhimento e vínculo, entre os pacientes, seus acompanhantes e os serviços de saúde. 0 processo de educação e de confiabilidade pode estimular aos pacientes a responsabilidade do autocuidado, gerando a interpretação de que muitas situações são preveníveis. É nesse momento, que os profissionais de enfermagem têm a oportunidade de detectar problemas de saúde, que podem ser expressados através das falas ou de expressões 
corporais e faciais dos pacientes como, por exemplo, os sinais e sintomas da dor. A partir desse contato, é possível também aos profissionais tirar dúvidas e promover a educação em saúde, sendo assim, possível compreender essa clientela de forma integral e humanizada atendendo aos princípios do Sistema Único de Saúde. ${ }^{9}$

Diante disso, este estudo objetivou relatar as experiências vivenciadas por uma enfermeira e sua equipe de enfermagem, referente ao atendimento acolhedor prestado aos pacientes e seus familiares em um ambulatório de oncologia clínica de um hospital especializado na cidade do Rio de Janeiro.

\section{METODOLOGIA}

Trata-se de um relato de experiência, descrito por uma enfermeira que atua em um ambulatório de oncologia clinica e hematologia de um hospital especializado, referente ao atendimento prestado pela mesma e sua equipe de enfermagem.

O ambulatório de oncologia clínica e hematologia realizam atendimentos de segunda a sexta feira, de 7 às 19 horas. Atende em média trezentos e cinquenta a quatrocentos pacientes por mês e conta com dez (10) ambulatórios de Oncologia Clínica. Um (1) consultório atende os pacientes que chegam ao setor apresentando intercorrências clínicas, os pacientes que necessitam ser avaliados pelo médico para checagem do exame de sangue para liberar a quimioterapia e os outros nove (9) consultórios, atendem os pacientes com consultas previamente agendadas.

O ambulatório de hematologia conta com três (3) consultórios, um (1) atende pacientes de consultas extras e dois (2) atendem pacientes com consultas marcadas. Temos também uma sala para realização de atendimentos e/ou procedimentos de enfermagem tais como: repouso, soroterapia, preparo e administração de medicamentos, realizações de curativos, entre outros. Os pacientes que necessitam de internação e estão apresentando alguma comorbidades ficam nesta sala aguardando a liberação do leito. Contamos também com uma sala de procedimentos médicos, onde são realizados: punção lombar, biópsia de medula óssea e mielograma. Todos esses fatores acabam desencadeando um grau de ansiedade e estresse elevado, não somente aos pacientes como aos seus acompanhantes.

$\mathrm{O}$ volume de atendimentos e procedimentos realizados no ambulatório, o estado de saúde mais comprometido dos pacientes e o grau de complexidade, requer dos profissionais médicos e de enfermagem um atendimento bastante criterioso. Todos esses fatores geram um tempo de espera maior, e, consequentemente, leva a ansiedade e ociosidade, não só aos pacientes, bem como aos acompanhantes.

O período de espera nos ambulatórios é momento de angústia e ansiedade, um tempo que poderia ser melhor aproveitado, pois nesse período de permanência na sala de espera, os pacientes e seus familiares passam por medos e incertezas em relação aos resultados de exames, atendimentos e tantas outras situações que geram expectativas em relação ao tratamento.

Diante disso, evidenciou-se a importância do acolhimento aos pacientes e familiares, no ambulatório de oncologia clínica e como uma grande contribuição para o serviço foi implantado o "Projeto Sala de Espera" no ambulatório de oncologia clínica e hematologia: Otimizando o tempo dos pacientes acompanhantes e fortalecendo, assim, o manejo e atendimento no ambulatório de oncologia clínica.

\section{RESULTADOS E DISCUSSÃO}

O ambulatório de oncologia clínica tem uma característica diferenciada aos outros, pois os pacientes atendidos, em sua maioria encontram-se em situações de complicações, que giram em torno das reações adversas ao tratamento ou ao avanço do processo patológico. Estes apresentam condições clínicas ruins e importantes comorbidades como: debilidades físicas, comprometimentos hemodinâmicos, além do medo, da ansiedade e muitas dúvidas. Todas essas condições podem levar o paciente a apresentar alguma intercorrência a qualquer momento do período em que o mesmo aguarda 0 atendimento.

Vale ressaltar que as repercussões supracitadas podem acontecer na recepção, sala de observação e/ou sala de procedimentos, por isso, a equipe multiprofissional, deve estar sempre atenta e preparada para atuar em diversas situações.

O primeiro contato dos pacientes e seus familiares no ambulatório acontece na recepção, no momento da entrega do cartão de consulta ou dos encaminhamentos, para os pacientes de primeira vez. As recepcionistas são profissionais bastante comprometidos com o serviço e realizam um atendimento de qualidade, estes possuem uma sintonia importante com os profissionais de enfermagem. Assim quando o paciente chega à recepção com qualquer queixa, imediatamente a equipe de enfermagem recebe a informação da necessidade do mesmo e realiza o atendimento.

O paciente e seu familiar são direcionados à sala de enfermagem, sendo recebido pelo enfermeiro e o técnico de enfermagem, onde são aferidos os sinais vitais. 0 enfermeiro realiza a avaliação a partir do exame físico associado ao diálogo, a fim de identificar as necessidades de saúde e de possível necessidade de prioridade para o atendimento médico a partir das queixas descritas pelo paciente e/ou familiar.

A assistência de enfermagem não está voltada apenas para solucionar os problemas clínicos e imediatos, mas, sim em realizar um atendimento holístico visando atender as necessidades integrais de saúde do cliente e seus familiares. 
A equipe de enfermagem, fornece as orientações educativas no que se refere a realização de vários procedimentos específicos ao tratamento no qual o paciente esteja sendo submetido no período tais como: administração de medicamento por via subcutânea, por via oral, retal, realizações dos curativos das lesões cutâneas, o cuidado com a integridade da pele, enfim, vários são os procedimentos e cuidados que o paciente oncológico realiza no cenário domiciliar. A equipe de enfermagem em cumplicidade com a equipe interdisciplinar em saúde elaborou estratégias para contribuir com a identificação e o manejo das principais repercussões hemodinâmicas, favorecendo um equilíbrio de estabilidade.

A ação profissional da enfermeira oncológica engloba uma grande diversidade de atividades, com variados graus de complexidade, cabendo-lhe igual empenho na realização de qualquer uma delas, tais como o atendimento aos clientes na recepção ou porta de entrada, resolutividade nas intercorrências, bem como habilidades e competências na tomada de decisão. ${ }^{10}$

Diante disso, face às situações de urgência e emergência na rotina, percebe-se que as principais complicações giram em torno das reações adversas ao tratamento ou ao avanço do processo patológico, podendo apontar como as principais: dor, febre, náuseas, vômitos, diarreias, constipações, crises convulsivas, lipotímias, hipotensões e raramente parada cardiorrespiratória.

Algumas das intercorrências supracitadas levam os profissionais de enfermagem a um pensamento crítico reflexivo sobre o atendimento ambulatorial, no que se refere a situações inusitadas, inesperadas, conflitantes que podem surgir no período em que os pacientes aguardam ser chamados para o atendimento médico.

Sabe-se que por muitas vezes os acompanhantes e profissionais não estão preparados habitualmente para lidar com essas intercorrências em um ambiente ambulatorial.

Essas situações fortalecem ainda mais a necessidade do acolhimento holístico e humanizado, entendendo que a ordem de chegada nem sempre será a única prioridade a ser avaliada, mas a possibilidade de um olhar clínico direcionado para investigação de possíveis instabilidades hemodinâmicas, podendo então dar ao profissional de saúde o pensamento crítico para tomada de decisão. No ambulatorial, os pacientes instáveis ou com possibilidade de instabilidade são atendidos prioritariamente, ou são levados para a sala de observação, onde a equipe de enfermagem de forma holística atua efetivamente para restaurar as funções corporais do paciente. Avaliar os riscos e a vulnerabilidade implica estar atento tanto ao grau de sofrimento físico quanto psíquico, pois muitas vezes o usuário que chega andando, sem sinais visíveis de problemas físicos, mas muito angustiado, pode estar mais necessitado de atendimento e com maior grau de risco e vulnerabilidade do que outros pacientes aparentemente mais necessitados. ${ }^{1}$
O acolhimento é realizado com orientações direcionadas aos pacientes e familiares, referente aos processos fisiopatológicos, das possibilidades de instabilidades ou repercussões hemodinâmicas previsíveis, fortalecendo assim as habilidades do autocuidado e de uma assistência de qualidade, estimulando o empoderamento do conhecimento e um cuidar holístico, além do funcionamento e fluxo do atendimento no ambulatório.

Nesse momento aproveita-se para estreitar a relação, pois acredita-se que essa ação seja capaz de promover a confiança entre pacientes, familiares e os profissionais, bem como fortalecer seus valores e crenças na possibilidade de eficácia e maior adesão ao tratamento.

Esta estratégia de um atendimento mais acolhedor, realizada pela equipe interdisciplinar, garantiu a qualidade da assistência prestada aos pacientes e seus familiares, com a prevenção das reações inesperadas no período de espera para o atendimento médico, bem como maior adesão ao tratamento e a continuidade do cuidado em domicílio.

O cuidado humanizado significa tornar humano, dar condições humanas a pessoa que esta necessitando de cuidados, não de forma técnica, mecanizada, mas como processo vivencial, sendo assim a relação do profissional de enfermagem com o paciente oncológico e seus familiares, no ambulatório de oncologia clínica e hematologia, busca promover segurança e bem-estar tanto individual quanto coletivo. ${ }^{11}$

\section{CONCLUSÃO}

As experiências vivenciadas no ambulatório de oncologia clínica contribuíram de formas satisfatórias na compreensão de gerenciar os serviços e as ações da equipe de enfermagem, no que se refere acolhimento aos pacientes e familiares, pois permitiu apontar estratégias que facilitaram a logística de atendimento, contribuindo para uma assistência de qualidade, humanizada, integral e eficaz.

Assim, a equipe interdisciplinar, necessita de uma comunicação terapêutica eficiente entre profissionalpaciente/familiares e monitorização constante das repercussões hemodinâmicas enquanto os pacientes aguardam atendimento, visto que as instabilidades acontecem de forma imprevisível.

Portanto, as contribuições do estudo vão além do contexto humanístico, mas transcendem a uma avaliação clínica para tomada de decisão, onde neste contexto oncológico o tempo pode ser crucial para uma assistência integral que possa garantir a vida e/ou a qualidade de vida, favorecendo dessa forma um acolhimento baseado nos princípios norteadores das políticas públicas de saúde.

Frente às lacunas nos modelos de atenção e gestão dos serviços de saúde pública, no que se refere ao acesso e ao modo como o usuário é acolhido, sugere-se estudos que destaquem a abordagem do cuidado humanizado 
e acolhedor aos pacientes oncológicos e seus familiares em atendimento ambulatorial, possibilitando o acesso ao conhecimento necessário à sua situação de saúde, em busca de promoção da autonomia para o autocuidado, bem como da qualidade de vida.

\section{REFERÊNCIAS}

1. Ministério da Saúde (BR). Secretaria de Atenção à Saúde. Núcleo Técnico da Política Nacional de Humanização. Acolhimento nas práticas de produção de saúde. Brasília (DF): MS; 2010.

2. Ministério da Saúde (BR) - Instituto Nacional de Câncer José Alencar Gomes da Silva. Coordenação de Prevenção e Vigilância. Estimativa 2014: Incidência de Câncer no Brasil/ Instituto Nacional de Câncer José Alencar Gomes da Silva, Coordenação de Prevenção e Vigilância. Rio de Janeiro: INCA, 2014.

3. Sistema de regulação de vagas - SISREG - Data SUS - Manoal operador solicitante. SISREG III solicitação de agendamento de consultas e procedimentos - Brasil setembro - 2008.

4. Zillmer JGV, Schwartz E, Muniz RM.O olhar da enfermagem sobre as práticas de cuidado de famílias rurais à pessoa com câncer. Rev. esc. enferm. USP [Internet]. 2012, 4(6):1371-1378. ISSN 0080-6234. Disponível em: http://dx.doi.org/10.1590/ S0080-62342012000600013.

5. Guerrero GP; Zago MMF, Sawada NO, Pinto MH. Relação entre espiritualidade e câncer: perspectiva do paciente. Revista Brasileira de Enfermagem [Internet]. 2011, 64(1):5359. Disponível em: http://www.scielo.br/pdf/reben/v64n1/ v64n1a08.pdf.

6. Salimena AMO, Teixeira SR, Amorim TV. O vivido dos enfermeiros no cuidado ao paciente oncológico. Cogitare Enferm [Internet]. 2013 Jan/Mar; 18(1):142-7. Disponível em: http://revistas.ufpr.br/cogitare/article/view/31320.

7. Silva RCV, Cruz EA. Planejamento da assistência de enfermagem ao paciente com câncer: reflexão teórica sobre as dimensões sociais. Esc. Anna Nery [Internet]. 2011, 15(1):180-185. ISSN 1414-8145. Disponível em: http:// dx.doi.org/10.1590/S1414-81452011000100025.

8. Reis FV, Brito JR, Santos JN, Oliveira MG. Educação em saúde na sala de espera - relato de experiência Educação em saúde na sala de espera - relato de experiência. Rev Med Minas Gerais [Internet]. 2014; 24(1): s32-s36. Disponível em: rmmg.org/exportar-pdf/549/v24s1a05.pdf.

9. Carneiro ACLL, Souza V, Godinho LK, Faria ICM, Silva KL, Gazzinelli MF. Educação para a promoção da saúde no contexto da atenção primária. Rev Panam Salud Publica [Internet]. 2012; 31(2):115-20. Disponível: http://www. scielosp.org/pdf/rpsp/v31n2/a04v31n2.pdf.

10. Machado GC; Ouro ETR; Santana FPG. Acolhimento de enfermagem em oncologia: enfrentamento da doença pelos pacientes em Feira de Santana-BA. Revista Brasileira de Saúde Funcional, [Internet]. 2015; 2(1):33-45. Disponível em: http://www.seer-adventista.com.br/ojs/index.php/RBSF/ article/view/696.

11. Oliveira NES, Oliveira LMAC, Lucchese R, Alvarenga GC, Brasil V. Humanização na teoria e na prática: a construção do agir de uma equipe de enfermeiros. Rev. Eletr. Enf. [Internet]. 2013 abr/jun; 15(2):334-43. Disponível em: http://dx.doi. org/10.5216/ree.v15i2.17916. 